\title{
Management of Urinary Tract Infections in Direct to Consumer Telemedicine
}

\author{
Radhika Rastogi, $A B^{7}$, Kathryn A. Martinez, $P h D, M P H^{2}$, Niyati Gupta, $M D^{2}$, \\ Mark Rood, $\mathrm{MD}^{3}$, and Michael B. Rothberg, $M D, M P H^{2}$
}

${ }^{1}$ Cleveland Clinic Lerner College of Medicine of Case Western Reserve University , Cleveland, $\mathrm{OH}$, USA; ${ }^{2}$ Cleveland Clinic Center for Value-Based Care Research, Cleveland, OH , USA; ${ }^{3}$ Department of Family Medicine, Cleveland Clinic , Cleveland, OH , USA.

\begin{abstract}
BACKGROUND: Urinary tract infections (UTI) are a common reason for seeking care via direct to consumer (DTC) telemedicine, yet patterns of care, including antibiotic prescribing, have not been reported.
\end{abstract}

OBJECTIVE: To describe management of UTI in a large nationwide DTC telemedicine platform.

DESIGN: Cross-sectional observational study.

PARTICIPANTS: Patients seeking care for or diagnosed with UTI via DTC telemedicine between July 2016 and July 2018.

MAIN MEASURES: Patient measures included age, sex, geographic region, satisfaction with care, and patientreported call reason. High-risk patients were defined as males, patients over 65 years, or those diagnosed with pyelonephritis. Physician measures included specialty and geographic region. Antibiotic prescription was assessed overall and by antibiotic type. Variation in antibiotic prescriptions was assessed by patient and physician factors, including geographic region of both parties.

KEY RESULTS: Of the 20,600 patients diagnosed with a UTI during the study period, 96\% were female. Most (84\%) stated their call reason was a UTI. Overall, 94\% of UTI patients received an antibiotic; 56\% got nitrofurantoin, 29\% got trimethoprim-sulfamethoxazole, and 10\% got a quinolone. Receipt of an antibiotic was associated with higher satisfaction with care $(p<0.001)$. While nitrofurantoin was the most common antibiotic for all physician regions, antibiotic type varied by physician region. Of the $6 \%$ of the study population defined as high risk, 69\% received an antibiotic: $72 \%$ of males, $91 \%$ of women over 65 , and $21 \%$ of patients diagnosed with pyelonephritis.

CONCLUSIONS: Management of UTI via DTC telemedicine appears to be appropriate for average-risk patients, and most are able to self-diagnose. Most patients received guideline-concordant care, but over half of high-risk patients received antibiotics. DTC telemedicine offers convenient, low-cost care that is generally appropriate. Efforts should be made to ensure high-risk patients get proper follow-up.

$\mathrm{J}$ Gen Intern Med 35(3):643-8

DOI: $10.1007 / \mathrm{s} 11606-019-05415-7$

(๑) Society of General Internal Medicine 2019

Received February 27, 2019

Revised July 16, 2019

Accepted July 31, 2019

Published online October 30, 2019

\section{BACKGROUND}

Over $50 \%$ of women will experience a urinary tract infection (UTI) in their lifetime. ${ }^{1,2}$ As UTIs are often painful, ${ }^{3}$ they are a common reason to seek care, resulting in over 8 million outpatient visits and 1 million ED visits in the USA annually. Healthcare costs associated with UTI are estimated at over \$2 billion per year. ${ }^{1,2,4}$

Diagnoses of UTI can be made based on patient symptoms and history, and generally without need for a urine culture. ${ }^{5,6}$ Among nonpregnant, healthy, young women, treatment of uncomplicated UTI with empiric antibiotics is cost effective and reduces symptom duration. ${ }^{7-9}$ Additionally, recurrent UTI is common, and patients can safely identify and initiate treatment themselves. ${ }^{10,11}$ The Infectious Disease Society of America recommends treatment of uncomplicated UTI with nitrofurantoin or, if local resistance is lower than $20 \%$, trimethoprim-sulfamethoxazole (TMP-SMX).

Direct to consumer (DTC) telemedicine, or telemedicine services that are not affiliated with any particular health system or insurance plan, is increasingly common. ${ }^{12,13}$ In DTC telemedicine, patients can access care by licensed physicians 24 hours a day from their smart phone or tablet, and insurance is accepted but not required. DTC telemedicine serves as a substitute for urgent care or outpatient visits, where UTI patients frequently seek care. ${ }^{14}$

Studies evaluating telephone diagnosis and treatment of UTI have found that remote management of uncomplicated UTIs seems appropriate for most patients. ${ }^{15-19}$ Two such studies found high rates of antibiotic prescribing ${ }^{20}$ and low rates of urine culture referral. ${ }^{21}$ One of these used standardized patients, ${ }^{21}$ and therefore may not reflect patients seeking care in the realworld. The other was limited in size and geographic scope and only included insured, low-risk (e.g., not pregnant or elderly) patients from a single health system. ${ }^{20}$ To date, no study has assessed management of UTIs in DTC telemedicine. Given that most patients with UTI symptoms will seek medical care, and the lower cost of delivering care via telemedicine relative to brick and mortar settings, encouraging UTI management via telemedicine may be cost saving. However, the lack of urinalysis and culture raises the possibility of inappropriate care, particularly for those with complicated infections. Moreover, DTC telemedicine is unique in that it pairs patients with 
physicians from different parts of the country and does not explicitly exclude high-risk patients. Given antibiotic resistance patterns vary by geography, treatment decisions should take into account patient location.

The objective of this study was to describe UTI patients, encounters, and antibiotic prescribing in a large nationwide DTC telemedicine service. We also aimed to assess appropriateness of treatment based on patient risk level and geographic region.

\section{METHODS}

This study describes encounters between July 2016 and July 2018 on a large, national, direct-to-consumer telemedicine system. The telemedicine system provided their data to the authors for academic purposes, and had no role in the design, implementation, or publication of the study. This study was approved by the Cleveland Clinic's Institutional Review Board.

\section{Participants}

We included adult patients whose stated call reason was UTI or who were diagnosed with a UTI. Call reasons were determined based on free-text field patients can fill out to state their care-seeking reason. We classified patients as calling for UTI if their call reason contained variants of the words "urinary tract infection," "bladder infection" or "kidney infection". Patients indicating they were calling about symptoms of a UTI only (e.g., painful urination) were not considered to have stated their call reason was UTI.

Physicians recorded ICD-10 diagnosis codes associated with encounters. We included all codes associated with bacterial infections of the urethra, urinary tract, or bladder as UTI. The codes included were: N10, N30, N30.0, N30.00, N30.01, N30.10, N30.20, N30.80, N30.81, N30.90, N30.91, N34, N34.1, N34.2, N39.0, N39.9, and R30.0. We also considered patients who were diagnosed with dysuria and given antibiotics as being diagnosed with UTI because some telemedicine providers, in the absence of a urinalysis, may feel they cannot technically make a UTI diagnosis. In these cases, they diagnose what they feel they can (dysuria), but nonetheless treat the patient for UTI.

\section{Patient and Physician Characteristics}

We collected patient, physician, and encounter characteristics. Patients provided sex, age, insurance information, and state of residence, which we categorized in censusdefined regions. Insurance is not required to receive care and out-of-pocket cost was between $\$ 59$ and \$69 during the study period. We assumed patients who did not provide insurance information were not insured. Physician characteristics included physician specialty and geographic region, also categorized by census regions. Encounter characteristics included time of day and encounter length, which were recorded by the telemedicine system.

\section{Outcomes}

Our outcomes included antibiotic prescription and patient satisfaction. Antibiotic receipt was determined by National Drug Codes associated with the encounter. We categorized this both as yes/no and by antibiotic type (TMP-SMX, nitrofurantoin, quinolone, or other). Infectious Disease Society of America guidelines were used to designate antibiotics as appropriate or inappropriate. ${ }^{22}$ Appropriate regimens included nitrofurantoin, TMP-SMX, fosfomycin, and pivmecillinam. The guidelines recommend TMP-SMX be used only when local resistance is less than 20\%. Thus, in the absence of local resistance data, TMP-SMX could be inappropriate. All other antibiotics, including quinolones, were categorized as inappropriate. We also reported anesthetic prescriptions. Patient satisfaction with their telemedicine physician was assessed at encounter conclusion, rated on scales from 0 to 5 stars, with 5 being most satisfied. Consistent with treatment of other patient satisfaction measures (e.g., HCAHPS), we dichotomized this as "top box" or 5 vs. less than 5. Patients were also asked: "If you had not used online care today, where would you have gone instead?" Response categories included urgent care/retail clinic, doctor's office, emergency department, or done nothing.

\section{Statistical Analysis}

We generated descriptive statistics for the sample. We described variation in care by patient, encounter, and physician characteristics. Because of regional differences in antibiotic resistance patterns, the type of antibiotic prescribed should vary by patient geographic region. We assessed differences in antibiotic prescription patterns by patient and physician region, as well as patient risk level. We divided the sample into risk groups (average and high) based on patient age and sex. We considered women older than 65 years, males, and patients diagnosed with pyelonephritis to be high risk. While antibiotics in these patients might be appropriate, an in-person medical visit is warranted, and treatment through telemedicine alone is likely inappropriate. Statistical testing was done using chi-square tests or $t$ tests, as appropriate. All analyses were conducted in Stata 14.

\section{RESULTS}

\section{Patient and Encounter Characteristics}

The cohort consisted of 20,600 telemedicine patients diagnosed with a UTI between July 2016 and July 2018, and 84\% stated their call reason was UTI. Among the 18,850 patients who stated their call reason was a UTI, 92\% were diagnosed with UTI. Patient, physician, and encounter characteristics are presented in Table 1 . The majority of patients were female (96\%), and 53\% were between the ages of $18-39$ years, and $1 \%$ were 65 years and older. Most (34\%) were from the South, followed by the Midwest (31\%), West (22\%), and Northeast (12\%). Eighty- 
Table 1 Patient, Physician, and Encounter Characteristics

\begin{tabular}{|c|c|c|c|}
\hline & \multirow{2}{*}{$\begin{array}{l}\text { Overall } \\
N(\% *)\end{array}$} & \multirow{2}{*}{$\begin{array}{l}\text { Antibiotic } \\
\text { received } \\
N(\% * *)\end{array}$} & \multirow[b]{2}{*}{$\begin{array}{l}p \\
\text { value }\end{array}$} \\
\hline & & & \\
\hline Number of patients & 20,600 & 19,364 & \\
\hline \multicolumn{3}{|l|}{ Sex } & $<$ \\
\hline Female & $19,844(96)$ & $18,840(94)$ & 0.001 \\
\hline Male & $756(4)$ & $546(72)$ & \\
\hline \multicolumn{3}{|l|}{ Age (years) } & 0.017 \\
\hline $18-29$ & $4991(24)$ & 4693(94) & \\
\hline $30-39$ & $6056(29)$ & $5674(94)$ & \\
\hline $40-49$ & $4907(24)$ & $4649(95)$ & \\
\hline $50-64$ & $4371(21)$ & 4121(94) & \\
\hline 65 and over & $275(1)$ & $249(91)$ & \\
\hline \multicolumn{3}{|l|}{ Region } & 0.257 \\
\hline Northeast & $2571(12)$ & $2427(94)$ & \\
\hline Midwest & $6433(31)$ & $6078(94)$ & \\
\hline South & $7062(34)$ & $6635(94)$ & \\
\hline West & $4534(22)$ & 4246(94) & \\
\hline \multicolumn{3}{|c|}{ Reported insurance information } & $<$ \\
\hline Yes & $18,065(88)$ & $17,054(94)$ & 0.001 \\
\hline No & $2535(12)$ & $2332(92)$ & \\
\hline \multicolumn{3}{|l|}{ Risk level } & $<$ \\
\hline Average & $19,429(94)$ & $18,574(96)$ & 0.001 \\
\hline High & $1171(6)$ & $812(69)$ & \\
\hline \multicolumn{3}{|l|}{ Call time of day } & 0.120 \\
\hline $9 \mathrm{pm}$ to $7 \mathrm{am}$ & $3145(15)$ & $2983(95)$ & \\
\hline $7 \mathrm{am}$ to $5 \mathrm{pm}$ & $12,960(63)$ & $12,189(94)$ & \\
\hline $5 \mathrm{pm}$ to $9 \mathrm{pm}$ & $4495(22)$ & $4214(94)$ & \\
\hline \multicolumn{3}{|c|}{ Where they would have accessed care otherwise } & $<$ \\
\hline Doctor's office & $3702(26)$ & $3547(96)$ & 0.001 \\
\hline Emergency department & $506(4)$ & $475(94)$ & \\
\hline Urgent care & $9033(63)$ & $8644(96)$ & \\
\hline Done nothing & $1034(7)$ & $950(92)$ & \\
\hline \multicolumn{3}{|c|}{ Percent rating physician 5 stars } & \\
\hline No & $918(6)$ & $776(85)$ & 0.001 \\
\hline Yes & $14,147(94)$ & $13,550(96)$ & \\
\hline \multicolumn{3}{|l|}{ Physician specialty } & $<$ \\
\hline $\begin{array}{l}\text { Family medicine }(n= \\
355)\end{array}$ & $14,138(69)$ & $13,382(95)$ & 0.001 \\
\hline \multicolumn{4}{|l|}{ 176) } \\
\hline $\begin{array}{l}\text { Emergency medicine ( } n \\
=94)\end{array}$ & $1584(8)$ & $1496(94)$ & \\
\hline Other specialty or PA/ & $252(1)$ & $226(90)$ & \\
\hline \multicolumn{4}{|l|}{$\mathrm{NP}(n=56)$} \\
\hline \multicolumn{3}{|l|}{ Physician region } & 0.009 \\
\hline Northeast $(n=117)$ & $3953(19)$ & $3704(94)$ & \\
\hline Midwest $(n=127)$ & $5239(25)$ & $4980(95)$ & \\
\hline South $(n=288)$ & $7736(37)$ & $7252(94)$ & \\
\hline West $(n=149)$ & $3672(18)$ & $3450(94)$ & \\
\hline
\end{tabular}

eight percent reported insurance information. The majority of encounters (63\%) occurred between $7 \mathrm{am}$ and $5 \mathrm{pm}$, and $15 \%$ occurred between $9 \mathrm{pm}$ and $7 \mathrm{am}$. There were 681 physicians in the sample. Most patients were seen by family medicine physicians $(69 \%)$, followed by internal medicine $(22 \%)$ and emergency medicine $(8 \%)$ physicians.

Among the $69 \%$ of UTI patients who indicated what they would have done had they not used telemedicine that day, $63 \%$ reported they would have gone to urgent care, $26 \%$ to the doctor's office, and $4 \%$ to the ED; $7 \%$ would have done nothing. Between $9 \mathrm{pm}$ and $7 \mathrm{am}, 7 \%$ of patients reported they would have gone to the ED vs. $2 \%$ between $7 \mathrm{am}$ and $5 \mathrm{pm}(p<$ $0.001)$. Among patients diagnosed with pyelonephritis, $65 \%$ would have gone to urgent care, $16 \%$ would have done nothing, $12 \%$ would have gone to the doctor's office, and $7 \%$ would have gone to the ED.

\section{Treatment}

Overall, 94\% of UTI patients received an antibiotic and 15\% received an anesthetic. Men were less likely to receive antibiotics than were women ( $72 \%$ vs. $94 \%, p<0.001)$, and patients over 65 years received antibiotics at a slightly lower rate than 20-29-yearolds ( $91 \%$ vs. $94 \%, p<0.001)$. Ninety-six percent of patients who rated their care five stars received an antibiotic, compared to $85 \%$ who rated their care less than 5 stars $(p<0.001)$

Table 2 presents the variation in the type of antibiotic prescribed by patient and physician region. Most patients received guideline-concordant treatment. Overall, $56 \%$ of patients got nitrofurantoin, 29\% got TMP-SMX, $10 \%$ got a quinolone, and $4 \%$ got another antibiotic (e.g., ampicillin or amoxicillin). The choice of antibiotic varied by physician geographic region, with physicians from the West prescribing TMP-SMX at a higher rate than other regions. Antibiotic choice also varied by patient geographic region, in a pattern similar to that of physicians, but to a lesser extent. Of the 278 physicians who had at least 10 UTI encounters, $15 \%(n=42)$ prescribed a quinolone $30 \%$ or more of the time and were responsible for $43 \%$ for all quinolone prescriptions. Six physicians prescribed other antibiotics $30 \%$ or more of the time.

The high-risk sample included 744 male patients, 253 women 65 years or older, and 174 patients with pyelonephritis, comprising $6 \%$ of the study population overall. Sixty-nine percent of high-risk patients received an antibiotic, and of these, $29 \%$ received a quinolone, compared to $9 \%$ of average-risk patients $(p<0.001)$. Of the 34 patients with pyelonephritis who received antibiotics, $56 \%$ were treated with quinolones, $26 \%$ with TMP-SMX, and $3 \%$ nitrofurantoin.

Table 2 Variation in the Type of Antibiotic Prescribed

\begin{tabular}{|c|c|c|c|c|}
\hline & \multirow{2}{*}{$\frac{\text { Nitrofurantoin }}{N(\%)}$} & \multirow{2}{*}{$\frac{\text { TMP-SMX }}{N(\%)}$} & \multirow{2}{*}{$\frac{\text { Quinolone }}{N(\%)}$} & \multirow{2}{*}{$\begin{array}{l}\text { Other } \\
N(\%)\end{array}$} \\
\hline & & & & \\
\hline Overall & $10,950(56)$ & $5668(29)$ & 1971(10) & $797(4)$ \\
\hline \multicolumn{5}{|c|}{ Physician region } \\
\hline Northeast & $2334(63)$ & $877(24)$ & $389(10)$ & $104(3)$ \\
\hline Midwest & $2907(58)$ & $1440(29)$ & $432(9)$ & 201(4) \\
\hline South & $3970(55)$ & $2150(30)$ & $825(11)$ & $307(4)$ \\
\hline West & $1739(50)$ & 1201(35) & $325(10)$ & $185(5)$ \\
\hline \multicolumn{5}{|c|}{ Patient region } \\
\hline Northeast & $1485(61)$ & $612(25)$ & $240(10)$ & $90(4)$ \\
\hline Midwest & $3399(56)$ & $1875(31)$ & $581(9)$ & $223(4)$ \\
\hline South & $3811(57)$ & $1837(28)$ & $712(11)$ & $275(4)$ \\
\hline West & $2255(53)$ & $1344(32)$ & $438(10)$ & $209(5)$ \\
\hline \multicolumn{5}{|l|}{ Risk level } \\
\hline Average & $10,767(58)$ & $5417(29)$ & $1724(9)$ & $666(4)$ \\
\hline High & $183(23)$ & $251(31)$ & $247(30)$ & $131(16)$ \\
\hline
\end{tabular}




\section{DISCUSSION}

In our study of UTI visits in a large nationwide DTC telemedicine service, we found high concordance between patients' self-diagnosis and physician diagnosis. The majority of patients diagnosed with a UTI were prescribed an appropriate antibiotic, most frequently nitrofurantoin. Patient satisfaction with telemedicine care was high, and telemedicine appears to serve as a substitute for urgent care, and to a lesser extent, office visits. We also identified some potentially inappropriate care, as many high-risk patients - men, women over 65 , and patients diagnosed with pyelonephritis - received antibiotic prescriptions, and a few received prescriptions for nitrofurantoin. Physicians largely followed national guidelines for antibiotic choice, but there were a few outliers who prescribed quinolones or other antibiotics to a large proportion of their patients. Overall, DTC telemedicine appears to be an appropriate setting in which to treat UTI for average-risk patients.

Prior studies have investigated quality of care associated with telemedicine management of UTI. Schoenfeld and colleagues used standardized patients to assess UTI care via eight DTC telemedicine platforms. ${ }^{21}$ They found low adherence (34\% of visits) to guideline-indicated management of recurrent female UTI, though the real-world applicability of their study was limited by the use of a script by standardized patients and a small sample size. ${ }^{21}$ Another study in a single health system investigated safety of telephone management of UTI in a population that excluded patients based on high-risk symptoms. ${ }^{16}$ In this study, a triage nurse managing the calls could access the patients' complete medical records, and treatments were chosen from four predefined regimens. ${ }^{16}$ Several others have limited their populations to young, healthy, nonpregnant women. ${ }^{10,15,23,24}$ Our study, conducted in a nationwide DTC telemedicine platform, differs in several important ways. Patients in prior studies were clustered by geographic area or insurance plan ${ }^{16,20}$ or had to meet certain inclusion criteria, most frequently based on risk level. ${ }^{16}$ Our patients came from a diverse national sample, irrespective of insurance or clinical risk status. Our sample of telemedicine physicians did not have access to the patient's medical record and were not given guidance regarding recommended treatment. As a result, findings from our study are more likely to represent real-world care seeking and care delivery for UTI via large DTC telemedicine platforms, where UTI management is common.

National guidelines recommend nitrofurantoin as the firstline treatment for uncomplicated cystitis, and it was the most frequently prescribed antibiotic in our study. ${ }^{22,25}$ TMP-SMX is also acceptable if local resistance rates are less than $20 \%{ }^{22,25}$ Neither quinolones nor other antibiotics (such as $\beta$-lactams) are recommended as first-line treatment. A small percentage of patients, however, might be expected to receive them due to allergies or other specific circumstances. Though patients were prescribed quinolones $10 \%$ of the time in our cohort, a small portion of physicians prescribed quinolones more than $30 \%$ of the time, suggesting that inappropriate prescribing is limited to a few outliers. Overuse of quinolones is concerning given increasing resistance. ${ }^{26}$ Moreover, the Food and Drug Administration issued a safety communication warning about the risk of serious harms of quinolones (e.g., permanent peripheral neuropathy). ${ }^{26,27}$ Some physicians may therefore benefit from decision support, or simple treatment algorithms, to ensure appropriate antibiotic choice.

Pairing patients and physicians from different parts of the U.S. presents a unique challenge for antibiotic stewardship in nationwide DTC telemedicine platforms. The IDSA guidelines recommend tailoring treatments to local resistance levels, but because patients and physicians are often in different regions, physicians cannot apply local knowledge of resistance rates when treating patients in other parts of the country. In our sample, we examined whether physicians' decisions considered antibiotic resistance in the patient's location. We found that most antibiotic variation was driven by physician region, and did not reflect reported $E$. coli antibiotic resistance patterns. ${ }^{25}$ Despite the fact national rates of TMP-SMX resistance are reaching $30 \%$ (above the recommended threshold for TMP-SMX use), it was the second most commonly prescribed antibiotic in our study. That physicians projected local practices nationally was not surprising. We were unable to find contemporary estimates of TMP-SMX resistance by state or census region, ${ }^{28,29}$ suggesting it would virtually impossible for physicians to accurately gauge bacterial sensitivity in the patient's region. Moreover, usage of quinolones in our study was fairly consistent across regions, even though local resistance rates vary from 24 to $37 \% .^{25}$ Until better reporting of antibiotic resistance rates by region becomes available, DTC telemedicine providers should prescribe nitrofurantoin as firstline therapy.

Prior studies suggest that young women are at low risk for complications if treated via telemedicine. ${ }^{16,17,20,21,23}$ Bacterial entry into the urinary tract can occur easily due to the anatomical placement of the external genitourinary structures. ${ }^{30} \mathrm{In}$ contrast, the longer urethra in men and antibacterial activity in prostatic fluids makes uncomplicated UTIs in men less likely. ${ }^{31,32}$ Thus, a UTI in a male patient is frequently associated with compromised urinary flow secondary to structural or functional abnormalities. UTI management among the elderly is also not well delineated. Common comorbidities among the elderly, such as diabetes, can result in complications and worse outcomes with a UTI, and thus treatment without follow up may be concerning. ${ }^{33}$ Elderly patients are also at greater risk for adverse outcomes related to interactions between their antibiotics and existent medication regimen. ${ }^{34,35}$ Evidence for the safety of empiric treatment of UTI in these higher risk populations is limited. We found a high rate of antibiotic prescription for both groups. Guidelines recommend urine culture for all patients suspected of having pyelonephritis. ${ }^{22}$ Only quinolones are recommended empirically; nitrofurantoin, TMP-SMX, and B-lactams may be considered after pathogen sensitivity is known. ${ }^{22}$ Though most patients diagnosed with pyelonephritis were not given antibiotics, of 
those who were, almost half were prescribed an inappropriate regimen. Quality improvement initiatives in telemedicine should target treatment of high-risk groups as well as antibiotic choice.

A rich literature supports the safety telephone diagnosis of UTI and the accuracy of self-diagnosis. ${ }^{15,16,18,20,21}$ That $92 \%$ of patients in our sample who stated their call reason was UTI were diagnosed with a UTI fits with previous studies. Indeed, cost-effectiveness analyses suggest that empiric treatment based on symptoms, as described in our study, is the preferred strategy for young, healthy women. ${ }^{7,8}$ However, because physicians could not traditionally bill for this service, many are reluctant to provide it over the telephone, especially in the middle of the night, and may request patients visit their office in the morning or go to urgent care. The majority of our patients reported they would have sought care elsewhere had they not used telemedicine, particularly through doctor's offices or urgent care clinics. Relatively few indicated they would have visited an ED. Yet those who accessed the telemedicine system in the middle of the night - hours that urgent care settings are typically closed - were more likely to state they would have otherwise sought care at an ED. Management of UTI via DTC telemedicine might therefore have the greatest impact on urgent care utilization, with some potential impact on overnight ED use. Given the cost of providing telemedicine is lower than in-person care, increasing use of telemedicine for UTI management will undoubtedly reduce costs. ${ }^{20}$ The convenience and speed of diagnosis and treatment makes this a potentially ideal care setting for UTI management in averagerisk patients, and one that should appeal to health plans. In addition, $16 \%$ of those diagnosed with pyelonephritis stated they would not have sought care at all. By decreasing barriers to care, telemedicine may allow for prompt referral of patients with serious illness.

Our study has several limitations. We do not know what was discussed during the encounters, which limits our ability to understand the physicians' diagnostic or treatment approach. We also lack post-encounter follow-up data, so outcomes are unknown. For high-risk patients who received antibiotics, we do not know whether the telemedicine physician instructed them to seek in-person care in addition to initiating antibiotics. For some high-risk patients, this approach may have been appropriate, given individual circumstances. Additionally, while we found statistically significant differences in patterns of care by patient, physician, and encounter characteristics, many of these were small in absolute terms.

\section{CONCLUSION}

Management of UTIs via DTC telemedicine is appropriate for most average-risk female patients, and results in short encounters, high antibiotic prescribing rates as well as high patient satisfaction. Given the lower per-episode cost of telemedicine relative to traditional outpatient or urgent care, increasing management of UTIs through telemedicine is likely to be cost saving, and should be supported by health plans. While most antibiotic prescribing followed national guidelines, we identified several areas for improvement regarding prescribing to high-risk patients and choice of antibiotic prescribed.

Corresponding Author: Radhika Rastogi, Cleveland Clinic Lerner College of Medicine of Case Western Reserve University, 9500 Euclid Ave/NA21, Cleveland, $\mathrm{OH} 44195$, USA (e-mail: rastogr@ccf.org).

\section{Compliance with Ethical Standards:}

This study was approved by the Cleveland Clinic's Institutional Review Board.

Conflicts of Interest: The authors declare that they do not have a conflict of interest.

\section{REFERENCES}

1. Griebling TL. Urologic diseases in America project: trends in resource use for urinary tract infections in women. J Urol. 2005;173(4):12811287. doi:https://doi.org/10.1097/01.ju.0000155596.98780.82

2. Foxman B. Epidemiology of urinary tract infections: incidence, morbidity, and economic costs. Am J Med. 2002;113 Suppl:5S-13S. http:// www.ncbi.nlm.nih.gov/pubmed/12113866.

3. Ellis AK, Verma S. Quality of life in women with urinary tract infections: is benign disease a misnomer? J Am Board Fam Pract. 2000;13(6):392 397. http://www.ncbi.nlm.nih.gov/pubmed/11117334.

4. Desforges JF, Stamm WE, Hooton TM. Management of Urinary Tract Infections in Adults. N Engl J Med. 1993;329(18):1328-1334. doi:https://doi.org/10.1056/NEJM199310283291808

5. Bent S, Saint $\mathbf{S}$. The optimal use of diagnostic testing in women with acute uncomplicated cystitis. Am J Med. 2002;113 :20S-28S. http:// www.ncbi.nlm.nih.gov/pubmed/12113868.

6. Little $\mathbf{P}$, Moore $\mathbf{M} \mathbf{V}$, Turner $\mathbf{S}$, et al. Effectiveness of five different approaches in management of urinary tract infection: randomised controlled trial. BMJ. 2010;340:c199. http://www.ncbi.nlm.nih.gov/ pubmed/20139214.

7. Barry HC, Ebell MH, Hickner J. Evaluation of suspected urinary tract infection in ambulatory women: a cost-utility analysis of office-based strategies. J Fam Pract. 1997;44(1):49-60. http://www.ncbi.nlm.nih. gov/pubmed/9010371.

8. Fenwick EA, Briggs AH, Hawke CI. Management of urinary tract infection in general practice: a cost-effectiveness analysis. $\mathrm{Br} \mathrm{J}$ Gen Pract. 2000;50(457):635-639. https://www.ncbi.nlm.nih.gov/pmc/articles/PMC1313774/

9. Rothberg MB, Wong JB. All dysuria is local: A cost-effectiveness model for designing site-specific management algorithms. J Gen Intern Med. 2004;19(5):433-443. doi:https://doi.org/10.1111/j.1525-1497.2004.10440.x

10. Gupta K, Hooton TM, Roberts PL, Stamm WE. Patient-initiated treatment of uncomplicated recurrent urinary tract infections in young women. Ann Intern Med. 2001;135(1):9-16. http://www.ncbi.nlm.nih. gov/pubmed/11434727.

11. Glover M, Moreira CG, Sperandio V, Zimmern P. Recurrent urinary tract infections in healthy and nonpregnant women. Urol Sci. 2014;25(1):1-8. doi:https://doi.org/10.1016/j.urols.2013.11.007

12. Mehrotra A. The Convenience Revolution for Treatment of Low-Acuity Conditions. JAMA. 2013;310(1):35. doi:https://doi.org/10.1001/jama. 2013.6825

13. Japsen B. Doctors' Virtual Consults With Patients To Double By 2020 Forbes. 2015. https://www.forbes.com/sites/brucejapsen/2015/08/ 09/as-telehealth-booms-doctor-video-consults-to-double-by-2020/ \# 19bd64ca4f9b.

14. Martinez KA, Rood M, Jhangiani N, et al. Patterns of Use and Correlates of Patient Satisfaction with a Large Nationwide Direct to Consumer Telemedicine Service. J Gen Intern Med. 2018;33(10):1768-1773. doi:https://doi.org/10.1007/s11606-018-4621-5 
15. Barry HC, Hickner J, Ebell MH, Ettenhofer T. A randomized controlled trial of telephone management of suspected urinary tract infections in women. J Fam Pract. 2001;50(7):589-594. http://www.ncbi.nlm.nih. gov/pubmed/11485707.

16. Vinson DR, Quesenberry CP. The safety of telephone management of presumed cystitis in women. Arch Intern Med. 2004;164(9):1026-1029. doi:https://doi.org/10.1001/archinte.164.9.1026

17. Blozik E, Sommer-Meyer C, Cerezo M, von Overbeck J. Effectiveness and safety of telemedical management in uncomplicated urinary tract infections. J Telemed Telecare. 2011;17(2):78-82. doi:https://doi.org/10. $1258 /$ jtt.2010.100406

18. Schauberger CW, Merkitch KW, Prell AM. Acute cystitis in women: experience with a telephone-based algorithm. WMJ Off Publ State Med Soc Wisconsin. 2007;106(6):326-329. http://www.ncbi.nlm.nih.gov/ pubmed/17970014.

19. Saint S, Scholes D, Fihn SD, Farrell RG, Stamm WE. The effectiveness of a clinical practice guideline for the management of presumed uncomplicated urinary tract infection in women. Am $\mathrm{J}$ Med. 1999; 106(6):636-641. http://www.ncbi.nlm.nih.gov/pubmed/ 10378621.

20. Mehrotra A, Paone S, Martich GD, Albert SM, Shevchik GJ. A Comparison of Care at E-visits and Physician Office Visits for Sinusitis and Urinary Tract Infection. JAMA Intern Med. 2013;173(1):72. doi:https://doi.org/10.1001/2013.jamainternmed.305

21. Schoenfeld AJ, Davies JM, Marafino BJ, et al. Variation in Quality of Urgent Health Care Provided During Commercial Virtual Visits. JAMA Intern Med. 2016;176(5):635. doi:https://doi.org/10.1001/ jamainternmed.2015.8248

22. Gupta K, Hooton TM, Naber KG, et al. International Clinical Practice Guidelines for the Treatment of Acute Uncomplicated Cystitis and Pyelonephritis in Women: A 2010 Update by the Infectious Diseases Society of America and the European Society for Microbiology and Infectious Diseases. Clin Infect Dis. 2011;52(5):e103-e120. doi:https:// doi.org/10.1093/cid/ciq257

23. Campbell J, Felver M, Kamarei S. "Telephone treatment" of uncomplicated acute cystitis. Cleve Clin J Med. 1999;66(8):495-501. http:// europepmc.org/abstract/med/10486997.

24. Stuart ME, Macuiba J, Heidrich F, Farrell RG, Braddick M, Etchison S. Successful implementation of an evidence-based clinical practice guideline: acute dysuria/urgency in adult women. HMO Pract. 1997;11(4):150-157. http://europepmc.org/abstract/med/10176516.

25. Morrill HJ, Morton JB, Caffrey AR, et al. Antimicrobial Resistance of Escherichia coli Urinary Isolates in the Veterans Affairs Health Care System. Antimicrob Agents Chemother. 2017;61(5). doi:10.1128/ AAC.02236-16
26. Commissioner O of the. Press Announcements - FDA updates warnings for fluoroquinolone antibiotics on risks of mental health and low blood sugar adverse reactions. 2018. https://www.fda.gov/newsevents/newsroom/pressannouncements/ucm612995.htm.

27. Research C for DE and. Drug Safety and Availability - FDA Drug Safety Communication: FDA updates warnings for oral and injectable fluoroquinolone antibiotics due to disabling side effects. 2018. https://www. fda.gov/Drugs/DrugSafety/ucm511530.htm.

28. Karlowsky JA, Jones ME, Thornsberry C, Critchley I, Kelly LJ, Sahm DF. Prevalence of antimicrobial resistance among urinary tract pathogens isolated from female outpatients across the US in 1999. Int $\mathrm{J}$ Antimicrob Agents. 2001;18(2):121-127. doi:https://doi.org/10.1016/S09248579(01)00369-7

29. Zhanel GG, Hisanaga TL, Laing NM, et al. Antibiotic resistance in Escherichia coli outpatient urinary isolates: final results from the North American Urinary Tract Infection Collaborative Alliance (NAUTICA). Int J Antimicrob Agents. 2006;27(6):468-475. doi:https://doi.org/10.1016/j. ijantimicag.2006.02.009

30. HICKLING DR, SUN T-T, WU X-R. Anatomy and Physiology of the Urinary Tract: Relation to Host Defense and Microbial Infection. Microbiol Spectr. 2015;3(4). doi:https://doi.org/10.1128/microbiolspec.UTI-00162012

31. Seminerio JL, Aggarwal G, Sweetser S. 26-Year-Old Man With Recurrent Urinary Tract Infections. Mayo Clin Proc. 2011;86(6):557-560. doi:https://doi.org/10.4065/mcp.2010.0600

32. Hummers-Pradier E, Ohse AM, Koch M, Heizmann WR, Kochen MM. Urinary tract infection in men. Int $\mathrm{J}$ Clin Pharmacol Ther. 2004;42(7):360-366. http://www.ncbi.nlm.nih.gov/pubmed/15605687.

33. Nitzan O, Elias M, Chazan B, Saliba W. Urinary tract infections in patients with type 2 diabetes mellitus: review of prevalence, diagnosis, and management. Diabetes, Metab Syndr Obes Targets Ther. 2015;8:129-136. doi:https://doi.org/10.2147/DMSO.S51792

34. Mody L, Juthani-Mehta M. Urinary Tract Infections in Older Women. JAMA. 2014;311(8):844-854. doi:https://doi.org/10.1001/jama.2014. 303

35. Crellin E, Mansfield KE, Leyrat C, et al. Trimethoprim use for urinary tract infection and risk of adverse outcomes in older patients: cohort study. BMJ. 2018;360:k341. doi:https://doi.org/10.1136/bmj.k341

Publisher's Note Springer Nature remains neutral with regard to jurisdictional claims in published maps and institutional affiliations. 